ORIGINAL ARTICLE

\title{
The burden of cholera in the slums of Kolkata, India: data from a prospective, community based study
}

\author{
D Sur, J L Deen, B Manna, S K Niyogi, A K Deb, S Kanungo, B L Sarkar, D R Kim, \\ M C Danovaro-Holliday, K Holliday, V K Gupta, M Ali, L von Seidlein, J D Clemens, \\ S K Bhattacharya
}

See end of article for authors' affiliations

Correspondence to: Dr S K Bhattacharya, National Institute of Cholera and Enteric Diseases, P-33 CIT Rd. Scheme - XM, Beliaghata, Kolkata 700-010, India; sujitkbhattacharya@ yahoo.com; bsujit@vsnl. net

Accepted 23 May 2005 Published Online First 26 July 2005
Aims: To conduct a prospective, community based study in an impoverished urban site in Kolkata (formerly Calcutta) in order to measure the burden of cholera, describe its epidemiology, and search for potential risk factors that could be addressed by public health strategies.

Methods: The study population was enumerated at the beginning and end of the study period. Surveillance through five field outposts and two referral hospitals for acute, watery, non-bloody diarrhoea was conducted from 1 May 2003 to 30 April 2004. Data and a stool sample for culture of Vibrio cholerae were collected from each patient. Treatment was provided in accordance with national guidelines.

Results: From 62329 individuals under surveillance, 3284 diarrhoea episodes were detected, of which $3276(99 \%)$ had a stool sample collected and $126(4 \%)$ were culture confirmed cholera. Nineteen (15\%) were children less than 2 years of age, $29(23 \%)$ had severe dehydration, and $48(38 \%)$ were hospitalised. Risk factors for cholera included a household member with cholera during the period of surveillance, young age, and lower educational level.

Conclusions: There was a substantial burden of cholera in Kolkata with risk factors not easily amenable to intervention. Young children bear the brunt not only of diarrhoeal diseases in general, but of cholera as well. Mass vaccination could be a potentially useful tool to prevent and control seasonal cholera in this community.
$\mathrm{S}$ ince antiquity, cholera has plagued residents of the Ganges and Bramaputra River deltas; the site from where six of the seven recorded pandemics had begun. ${ }^{1}$ The state of West Bengal in India, together with neighbouring Bangladesh, is considered by some experts as the "homeland of cholera". Environmental and socioeconomic factors ensure the persistence of the disease. Hospital based surveillance in Kolkata (formerly Calcutta), the capital of West Bengal, confirms the annual occurrence of cholera, which is detected from as early as March to as late as November each year. ${ }^{2}$

Cholera produces acute severely dehydrating diarrhoea, often requiring intravenous fluids. Thus despite the widespread availability of oral rehydration solution, cholera continues to be a serious public health problem with outbreaks easily overwhelming treatment facilities. Better understanding of the burden, epidemiology, and potential risk factors for cholera is needed to develop and implement new and creative approaches to eliminate this ancient scourge. We therefore undertook a prospective, community based study in Kolkata.

\section{METHODS}

\section{The study site and population}

Kolkata is the third largest city in India. With its 13 million residents packed into 1450 square kilometres, it is one of the world's most densely populated cities. The study site, 0.7 square kilometres in size, was already in maps from 1856 as an impoverished residential area known as Narkeldanga. ${ }^{3}$ Today, the study site encompasses bustees, which are legally recognised and registered slums. ${ }^{4}$ In the study area, the streets are narrow with little space between houses, piped municipal water supply is intermittent, and several households share one or two latrines and water taps. Most sewage is collected in open gutters which overflow when it rains. Kolkata has three seasons, the cool dry months from November to February, the hot dry period from March to May, and the monsoon season from June to October.
A baseline census of the study population was carried out in early 2003, followed by a second census one year later. Age, sex, and educational level of each individual, as well as household expenditure (as a surrogate for income) and health related behaviours were recorded. Each household had a mean of 1.5 rooms and a median number of 5 members (range 1-30). The median monthly household expenditure was 3000 Indian Rupees (INR) or 67 US\$. ${ }^{5}$ Each household and individual was assigned a unique identification number and household identification cards were distributed.

\section{Surveillance for diarrhoea}

Five project health outposts were set up in the field and two at the city's infectious diseases and children's hospitals, the main referral centres for diarrhoea. The five field outposts were open daily from 8 am to $8 \mathrm{pm}$ and the two hospital outposts were open 24 hours a day. Each outpost, operated by a physician and five assistants, offered free treatment for mild illnesses and stool culture for cholera. Free transport from the field to hospital was also provided when required.

Outpost based surveillance for acute, watery, non-bloody diarrhoea was started on 1 May 2003, after a pilot period of 10 days, and conducted for one year. For each consenting patient from the study population presenting with diarrhoea, a case report form describing medical history, physical examination findings, and management was completed and a rectal swab was obtained. Treatment was provided in accordance with national guidelines. The patient's unique identification number from his/her household card was recorded on the case report form. In the absence of the household card, the ID number was retrieved from the computerised census database using the patient's name and other identifiers. Cholera cases were followed up at home seven days after presentation to verify identification and to record clinical outcome. 
Table 1 Clinical characteristics of cholera cases, by age group from a study site in Kolkata, India, 1 May 2003 to 30 April 2004

\begin{tabular}{lccc}
\hline & $\begin{array}{l}\text { Cholera cases in } \\
\text { those less than 2 } \\
\text { years }(\mathbf{n}=19)\end{array}$ & $\begin{array}{l}\text { Cholera cases in } \\
\text { those 2 years and } \\
\text { older ( } \mathbf{n}=107)\end{array}$ & p value \\
\hline Indicator of severity & $9(47)$ & $56(52)$ & 0.69 \\
$\begin{array}{l}\text { Number (\%) with vomiting } \\
\text { pumber (\%) who were given/took antibiotics prior to }\end{array}$ & $4(25)$ & $11(11)$ & 0.12 \\
Number (\%) with severe dehydration & $4(21)$ & $25(23)$ & 1 \\
Number (\%) hospitalised & $8(42)$ & $40(37)$ & 0.7 \\
Number (\%) treated with antibiotics & $14(74)$ & $52(49)$ & 0.04 \\
\hline
\end{tabular}

Practitioners in the area were encouraged to refer patients with loose bowel movement to the outposts for stool culture. In addition, health workers visited each household in the study site once a month to find out about illnesses, hospitalisations, and deaths. The health workers encouraged consultation for loose bowel movement at the outposts. Verbal postmortem examinations were completed by a physician for all reported deaths. ${ }^{6}$

\section{Laboratory methods}

The rectal swabs were placed in Cary-Blair transport medium, kept at room temperature, and transported to the National Institute of Cholera and Enteric Diseases, Kolkata. From the Cary-Blair media, specimens were plated directly onto thiosulphate citrate bile salt sucrose (TCBS) agar (Eiken Chemical Company, Tokyo, Japan). The specimens were also incubated in alkaline peptone water $(\mathrm{pH} 8.6)$ for $6-8$ hours at $37^{\circ} \mathrm{C}$, then plated onto TCBS. After overnight incubation at $37^{\circ} \mathrm{C}$, suspected colonies on the TCBS plates were tested biochemically and confirmed by agglutination with polyvalent $\mathrm{Ol}$ and monovalent Ogawa and Inaba antisera (Difco Laboratories, Detroit, Michigan). Non-agglutinating strains were tested with antiserum to Vibrio cholerae O139 strain.

\section{Data management, definitions, and analysis}

The census and case report forms were double entered into custom made data entry programs using FoxPro software (Microsoft, Seattle, Washington, USA). The individual identification number was used to link the surveillance to the census data. Data management programs included error, range, and consistency check programs.

Satellite images of the study area were enhanced using an image processing software package (ERDAS Imagine, Atlanta, USA) to facilitate the digitisation of houses in the study area. A ground survey was conducted to link each household identification number to a GIS number, using methods similar to those described elsewhere. ${ }^{7}$

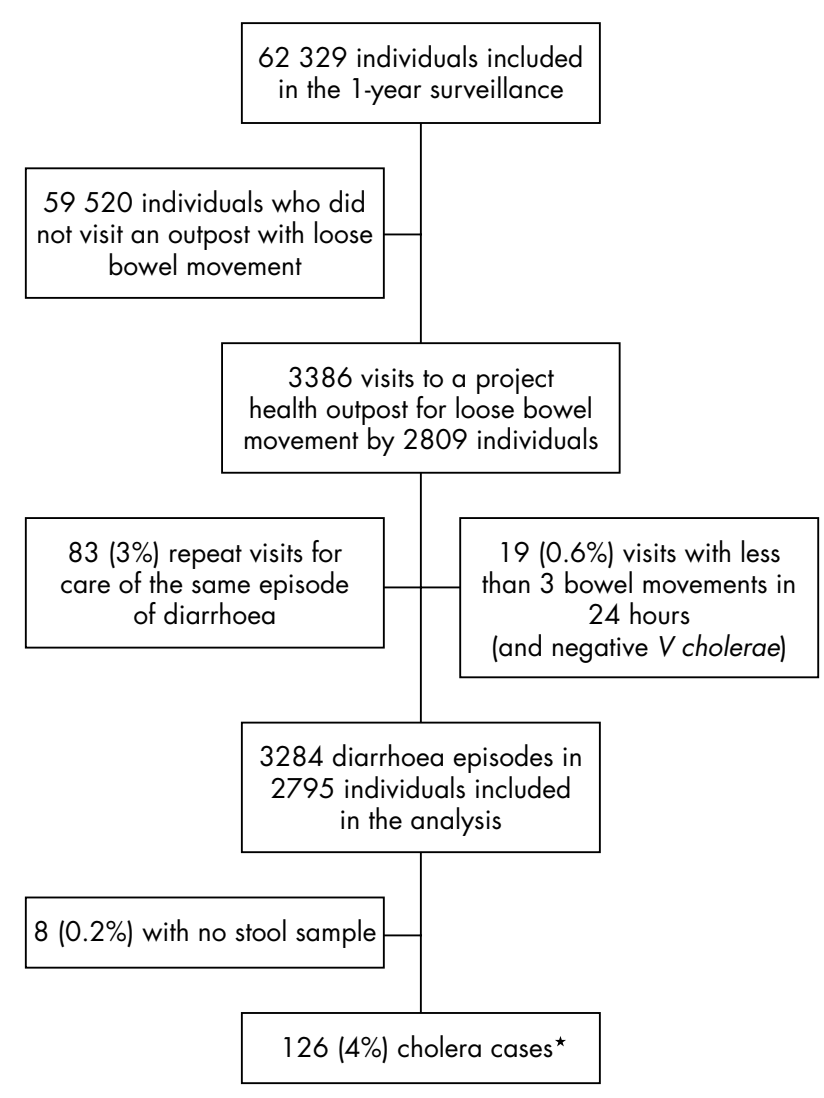

${ }^{\star}$ None of the cholera patients had a repeat episode of cholera.

Figure 1 Flow of patients visiting a project health outpost in a study site in Kolkata, India, 1 May 2003 to 30 April 2004.

Table 2 Antimicrobial resistance pattern of Vibrio cholerae $\mathrm{O} 1$ isolates from a study site in Kolkata, India, 1 May 2003 to 30 April 2004

\begin{tabular}{|c|c|c|c|c|c|c|}
\hline & \multicolumn{2}{|c|}{ Resistant } & \multicolumn{2}{|c|}{ Intermediate } & \multicolumn{2}{|c|}{ Susceptible } \\
\hline & No. & $\%$ & No. & $\%$ & No. & $\%$ \\
\hline Furazolidone $(n=77)$ & 66 & 86 & 5 & 7 & 6 & 8 \\
\hline Trimethoprim-sulphamethoxazole $(n=77)$ & 60 & 78 & 1 & 1 & 16 & 21 \\
\hline Erythromycin $(n=77)$ & 50 & 65 & 20 & 26 & 7 & 9 \\
\hline Tetracycline $(n=77)$ & 1 & 1 & 0 & 0 & 76 & 99 \\
\hline Doxycycline $(n=38)$ & 3 & 8 & 1 & 3 & 34 & 90 \\
\hline Ceftriaxone $(n=77)$ & 4 & 5 & 1 & 1 & 71 & 93 \\
\hline Ciprofloxacin $(n=77)$ & 6 & 8 & 8 & 10 & 63 & 82 \\
\hline Norfloxacin $(n=24)$ & 0 & 0 & 5 & 21 & 19 & 79 \\
\hline Azithromicin $(n=24)$ & 5 & 21 & 3 & 13 & 16 & 67 \\
\hline
\end{tabular}




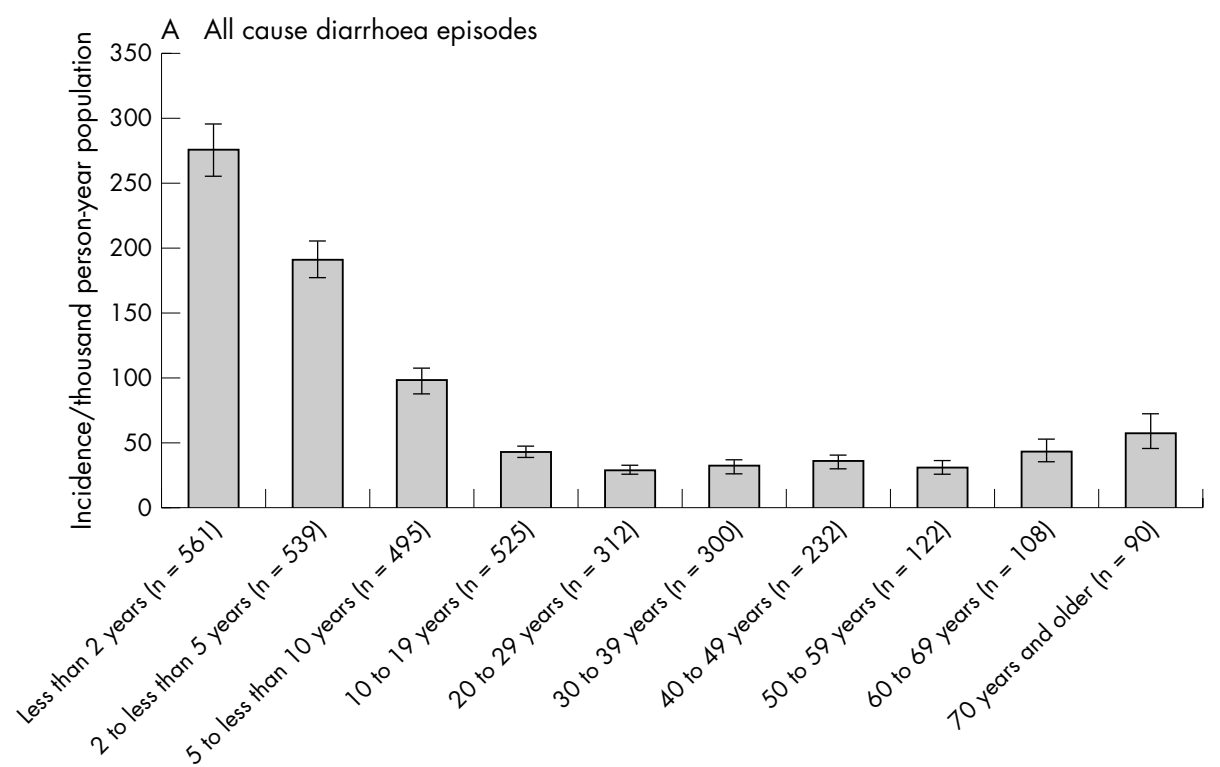

Age group

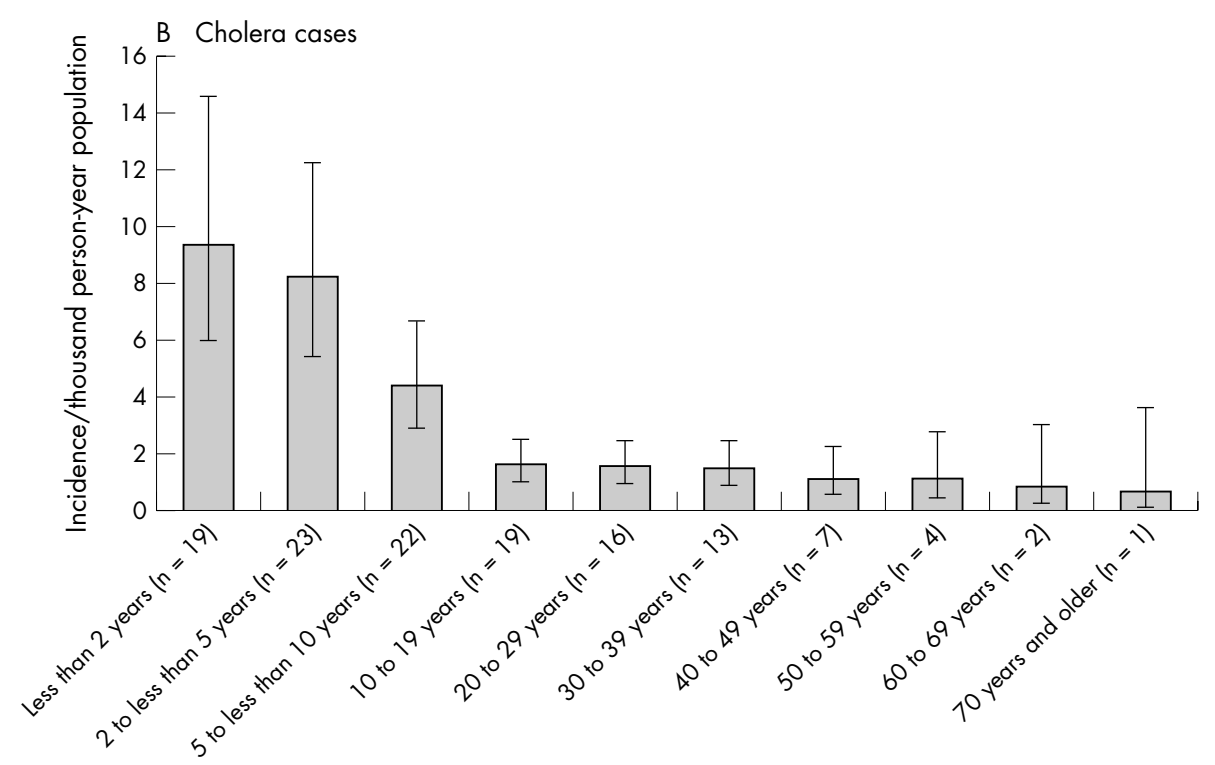

Age group

Figure 2 Age specific incidence rate (per 1000 person-years) of all cause diarrhoea episodes and cholera cases in a study site in Kolkata, India, 1 May 2003 to 30 April 2004.

A diarrhoea episode was defined as passage of three or more loose or liquid stools in 24 hours prior to presentation for care, which was considered the most practicable in children and adults. The onset of a diarrhoea episode was defined as the day on which it was reported to have begun. The end of a diarrhoea episode was defined as the day of hospital discharge or for outpatients, three days after presentation. A repeat visit was considered as a continuation of the previous episode if the end of the first episode and the onset of the second were three or fewer days apart. Cholera was defined as a diarrhoeal episode in which $V$ cholerae $\mathrm{Ol}$ or O139 was isolated in any component visit for that episode. A household was defined as a group of individuals residing in a geographically circumscribed area and recognising the same household head. Literacy was defined for those 15 years of age and older as having attended at least primary school.
The analysis was performed on a dynamic cohort. Age at onset of diarrhoea or cholera was used for age group categories. Person-time, the sum of person-years-at-risk contributed by each individual in the census, was used as the denominator in estimating the incidence rate of diarrhoea episodes and cholera cases. Person-time was calculated as follows: ${ }^{8}$

$$
p=\frac{\sum_{i=1}^{n}\left(e_{i}-s_{i}\right)-d_{i}}{x}
$$

where:

$e_{i}=$ end date of the surveillance period or date of exit of the $i$-th individual if exit from the study population occurred before the end date 
$s_{i}=$ start date of the study period or date of entry of the $i$-th individual if entry into the study population occurred after the start date

$d_{i}=$ duration (in days) of the diarrhoea or cholera illness of the $i$-th individual

$x=$ duration of the study period (in days)

The $95 \%$ confidence intervals of the incidence rates were calculated based on the Wilson score method. ${ }^{9}$

To assess for risk factors for cholera, we compared individual and household characteristics from the baseline census between cholera cases and individuals who did not visit an outpost for treatment of diarrhoea during the surveillance period. For both groups, we also analysed whether there was another member in the household with cholera detected at any time during the surveillance period. Unadjusted, crude odds ratios were calculated. To take into account that the risk of having cholera is likely to factor within a household, the odds ratios were recalculated by applying generalised estimating equations of the binomial family. ${ }^{10}$ We used an exchangeable form for the within-person correlation. The model was adjusted for having a household member with cholera during the surveillance period and age, variables which were found to be strongly associated with an increased risk for cholera in the univariate analysis.

All $\mathrm{p}$ values and $95 \%$ confidence intervals were interpreted in a two tailed fashion. Statistical significance was designated as a p value less than 0.05. Statistical analyses were performed using Stata 7 (Stata Corporation, USA) software.

\section{Ethics}

The study received approval from the Health Ministry Screening Committee of the Government of India and from the Secretariat Committee for Research Involving Human Subjects, World Health Organisation, Geneva, Switzerland.

\section{RESULTS}

From 1 May 2003 to 30 April 2004, a total of 62329 individuals were under surveillance for any period of time. There were 3386 visits for loose bowel movement from 2809 individuals from the study site (fig 1). After excluding 19 episodes $(0.6 \%)$ that did not fulfil the case definition of diarrhoea and $83(3 \%)$ repeat visits for care of the same episode of diarrhoea, 3284 diarrhoea episodes were included in the analysis. Of these, $3276(99 \%)$ had a stool sample collected and $126(4 \%)$ were culture confirmed as cholera.
During the one year of surveillance, there was no repeat cholera episode in any individual. Of the cholera cases, 19 (15\%) were children less than 2 years of age, 29 (23\%) had severe dehydration, 48 (38\%) were admitted to hospital, 66 $(52 \%)$ received antimicrobial therapy, and $1(0.8 \%)$ died. The death was in a child 3 years of age. Clinical characteristics were similar between those less than 2 years of age and older children and adults, except that young children were more frequently treated with antibiotics $(\mathrm{p}<0.05)$ (table 1$)$.

All of the isolates were identified as El Tor Ogawa $V$ cholerae O1. A total of $65-86 \%$ of isolates were resistant to the commonly used antimicrobials for cholera, furazolodone, trimethoprim-sulphamethoxazole, and erythromycin, but the majority remained susceptible to tetracycline and the quinolones (table 2).

With 56916 and 56958 person-years of observation, respectively, the overall incidence rate of treated diarrhoea episodes was 57.7 cases per thousand person-years (95\% CI 55.8 to 59.6) and of cholera was 2.2 cases per thousand person-years (95\% CI 1.9 to 2.6 ). When calculated by age group, we found that the incidence rate of cholera paralleled that of all-cause diarrhoeas (fig 2). The burden of cholera was greatest among those less than 2 years of age at 9.3 cases per thousand person-years (95\% CI 5.9 to 14.4). The youngest patient with culture proven cholera was 4 months of age.

During the surveillance year, cholera cases were detected during two seasons (fig 3). There were 51 cases from 1 May to 22 November 2003 and 75 from 29 March to 30 April 2004. Comparing the cholera cases detected during these two time periods, there was no difference in age distribution $(p>0.05)$ or geographic distribution of their households. Cases from both time periods were predominantly from the southwest corner of the study site, perhaps associated with higher population density in this area or proximity to water bodies (fig 4).

We compared characteristics between cholera cases and individuals who did not visit an outpost for treatment of diarrhoea during the surveillance period (table 3). Cholera cases were significantly more likely to have a household member with cholera, be less than 5 years of age, and have a lower educational level (for those over 15 years of age). There was also a trend for cholera cases to belong to households with an expenditure of less than 2000 INR per month and reporting unsafe health related behaviours such as irregular hand washing practices. We also compared characteristics

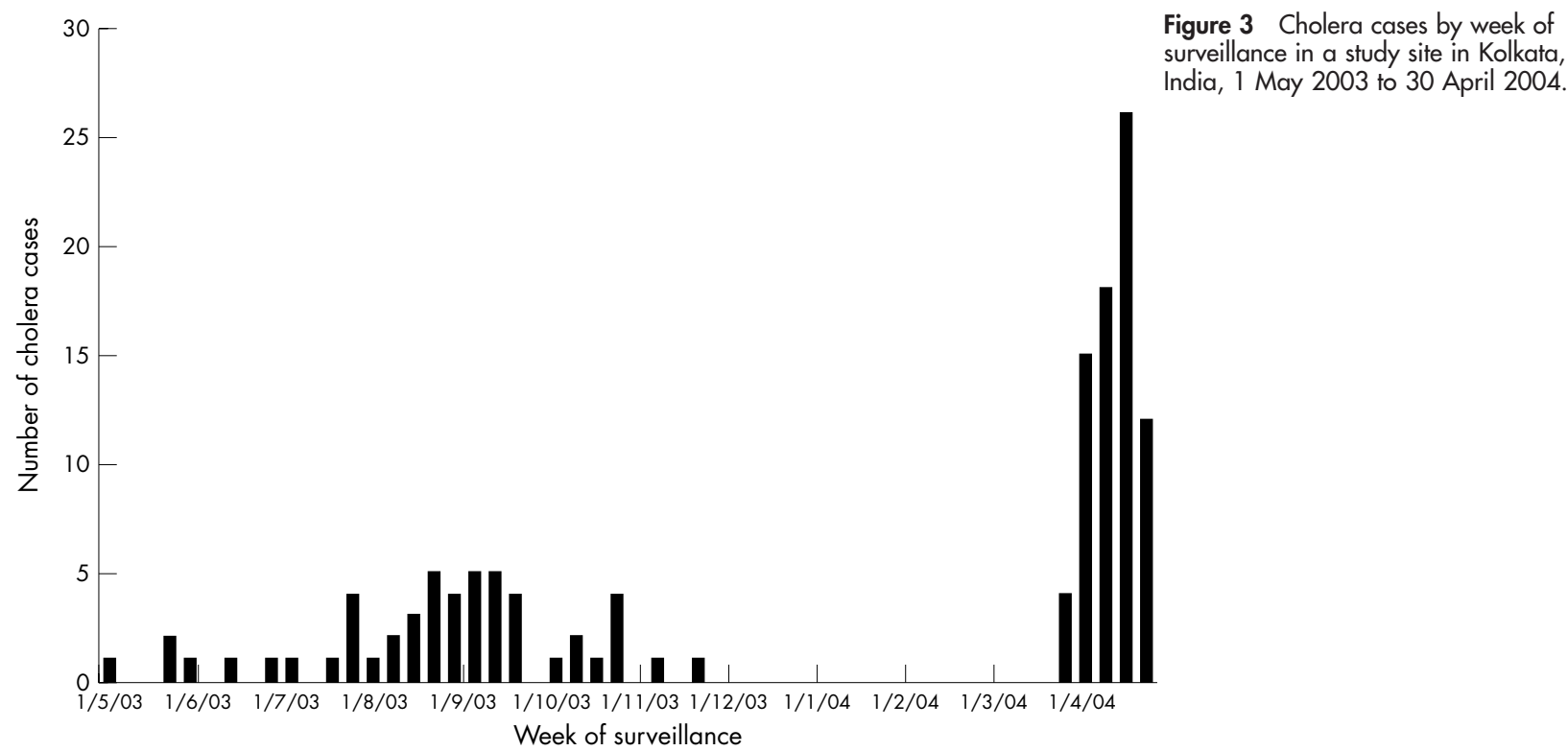

Figure 3 Cholera cases by week of surveillance in a study site in Kolkata, India, 1 May 2003 to 30 April 2004.

Week of surveillance 
Table 3 Characteristics of cholera cases compared with individuals who did not visit an outpost for diarrhoea during the surveillance period in a study site in Kolkata, India, 1 May 2003 to 30 April 2004

\begin{tabular}{|c|c|c|c|c|}
\hline No. $(\%)$ with the following characteristics & $\begin{array}{l}\text { Cholera cases } \\
(n=126)\end{array}$ & $\begin{array}{l}\text { Individuals with no } \\
\text { visit for diarrhoea } \\
\text { ( } n=59520 \text { ) }\end{array}$ & $\begin{array}{l}\text { Crude OR } \\
(95 \% \mathrm{Cl}), \mathrm{p} \text { value }\end{array}$ & $\begin{array}{l}\text { Adjusted* OR } \\
(95 \% \mathrm{Cl}), \mathrm{p} \text { value }\end{array}$ \\
\hline \multicolumn{5}{|l|}{ With other household member with cholera } \\
\hline None & $114(90)$ & $58799(99)$ & Ref & Ref \\
\hline One or more & $12(10)$ & 721 (1) & $8.6(4.7$ to 15.6$),<0.01$ & $8.1(4.4$ to 14.8$),<0.01 \dagger$ \\
\hline \multicolumn{5}{|l|}{ Age } \\
\hline$\geqslant 60$ months & $80(63)$ & 54772 (92) & Ref & Ref \\
\hline$<60$ months & $46(37)$ & $4748(8)$ & $6.6(4.6$ to 9.5$),<0.01$ & $6.6(4.6$ to 9.6$),<0.01$ \\
\hline \multicolumn{5}{|l|}{ Literacy of adults $\geqslant 15$ years old } \\
\hline Literate & $20(42)$ & $27751(65)$ & Ref & Ref \\
\hline Illiterate & $28(58)$ & $15265(35)$ & 2.5 (1.4 to 4.5$), 0.001$ & $2.5(1.4$ to 4.5$), 0.002$ \\
\hline \multicolumn{5}{|l|}{ Monthly household expenditure } \\
\hline$>44$ US $\$(2000$ INR) & $82(65)$ & $42746(73)$ & Ref & Ref \\
\hline$\leqslant 44$ US $\$(2000$ INR) & $44(35)$ & 16016 (27) & 1.4 (1.0 to 2.1$), 0.06$ & $1.4(1.0$ to 2.1$), 0.06$ \\
\hline \multicolumn{5}{|l|}{$\begin{array}{l}\text { Household members wash hands after } \\
\text { defecation }\end{array}$} \\
\hline Always & $79(63)$ & $40359(68)$ & Ref & Ref \\
\hline Sometimes or never & $47(37)$ & $19123(32)$ & $1.3(0.9$ to 1.8$), 0.2$ & $1.2(0.8$ to 1.7$), 0.4$ \\
\hline \multicolumn{5}{|l|}{ Type of house } \\
\hline Brick & $110(89)$ & $51937(88)$ & Ref & Ref \\
\hline Semi-brick/mud & $14(11)$ & $7238(12)$ & $0.9(0.5$ to 1.6$), 0.7$ & $0.9(0.5$ to 1.6$), 0.7$ \\
\hline \multicolumn{5}{|l|}{ Drinking water source } \\
\hline Bottled or tap & $124(98)$ & 58317 (98) & Ref & Ref \\
\hline Well, pump, or vendor & $2(2)$ & $1194(2)$ & $0.8(0.2$ to 3.2$), 0.7$ & $0.8(0.2$ to 3.6$), 0.8$ \\
\hline
\end{tabular}

between cholera cases and non-cholera diarrhoea cases (table 4). Cholera cases were significantly more likely to have a household member with cholera and have a lower educational level (for those over 15 years of age). Other characteristics were similar for both groups.

\section{DISCUSSION}

Our results show that cholera remains a persistent scourge in this community. The impact of the disease is aggravated by its severe manifestations and the frequent need for hospitalisation. Although young children bear the greatest burden of cholera, all age groups are affected. These findings are perhaps best explained by the early and frequent exposure to $V$ cholerae and recurrent susceptibility to the disease since naturally acquired immunity is not life long. ${ }^{11}$ There was only one cholera death during the surveillance year, but the case fatality rate is likely to be much higher under non-research conditions when immediate rehydration and transport to hospital may not be available.

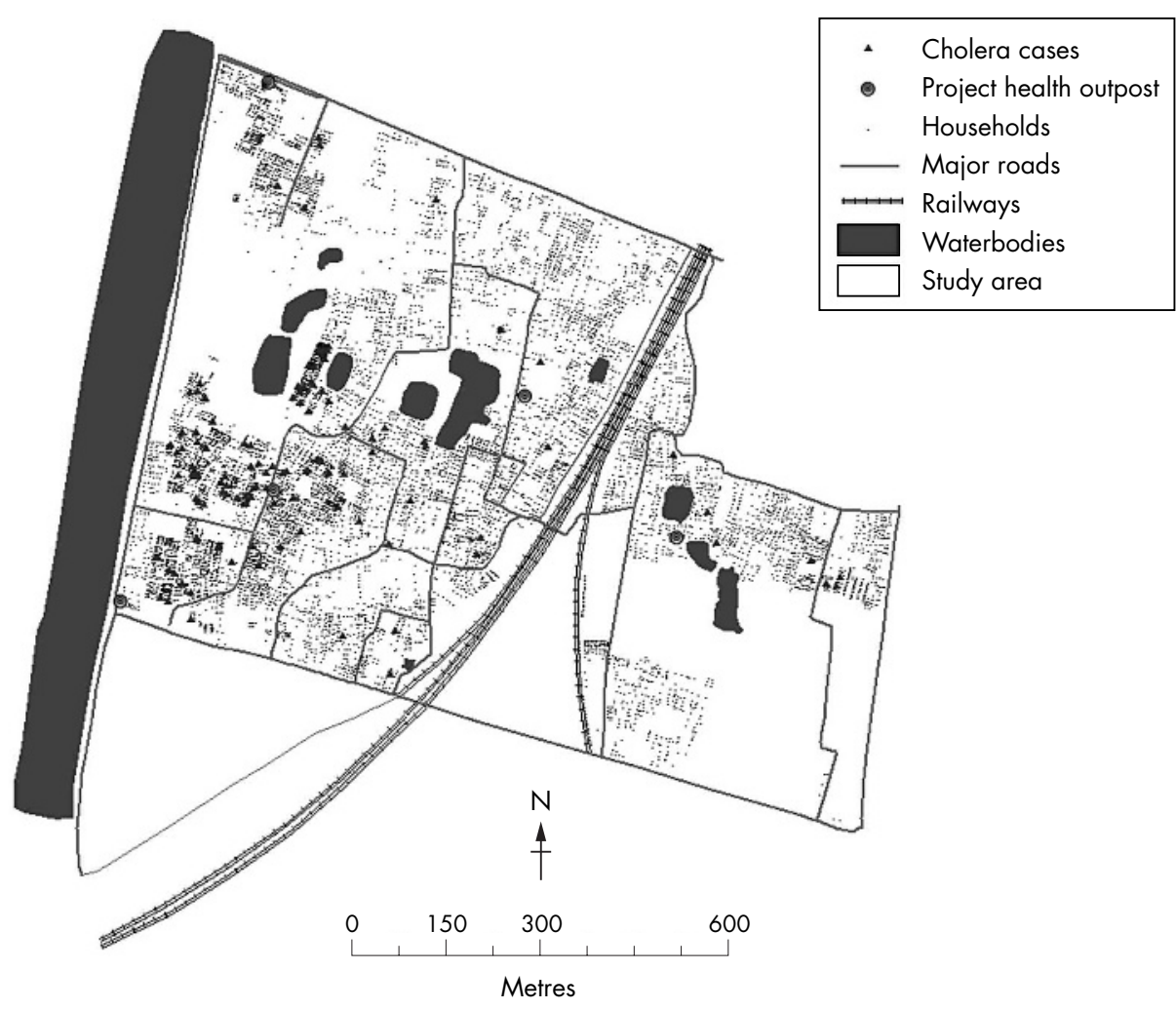

Figure 4 Geographic location of households of cholera cases in a study site in Kolkata, India, 1 May 2003 to 30 April 2004 
Table 4 Characteristics of cholera cases compared with non-cholera diarrhoea cases in a study site in Kolkata, India, 1 May 2003 to 30 April 2004

\begin{tabular}{|c|c|c|c|c|}
\hline No. $(\%)$ with the following characteristics & $\begin{array}{l}\text { Cholera cases } \\
(n=126)\end{array}$ & $\begin{array}{l}\text { Non-cholera } \\
\text { diarrhoea cases } \\
(n=2662 \text { ) }\end{array}$ & $\begin{array}{l}\text { Crude OR } \\
(95 \% \mathrm{CI}), \mathrm{p} \text { value }\end{array}$ & $\begin{array}{l}\text { Adjusted* OR } \\
(95 \% \mathrm{CI}), \mathrm{p} \text { value }\end{array}$ \\
\hline \multicolumn{5}{|l|}{ With other household member with cholera } \\
\hline None & $114(90)$ & $2573(97)$ & Ref & Ref \\
\hline One or more & $12(10)$ & 89 (3.3) & $3.0(1.6$ to 5.7$),<0.01$ & 3.1 (1.6 to 5.8$),<0.01 \dagger$ \\
\hline \multicolumn{5}{|l|}{ Age } \\
\hline$\geqslant 60$ months & $80(63)$ & $1841(69)$ & Ref & Ref \\
\hline$<60$ months & 46 (37) & $821(31)$ & $1.3(0.9$ to 1.9$), 0.18$ & $1.3(0.9$ to 1.9$), 0.15$ \\
\hline \multicolumn{5}{|l|}{ Literacy of adults $\geqslant 15$ years old } \\
\hline Literate & $20(42)$ & $682(58)$ & Ref & Ref \\
\hline Illiterate & $28(58)$ & 495 (42) & $1.9(1.1$ to 3.5$), 0.03$ & $1.9(1.1$ to 3.4$), 0.03$ \\
\hline \multicolumn{5}{|l|}{ Monthly household expenditure } \\
\hline$>44$ US\$ (2000 INR) & $82(65)$ & $1785(68)$ & Ref & Ref \\
\hline$\leqslant 44$ US\$ (2000 INR) & 44 (35) & 855 (32) & $1.1(0.8$ to 1.6$), 0.6$ & $1.1(0.8$ to 1.7$), 0.5$ \\
\hline \multicolumn{5}{|c|}{ Household members wash hands after defecation } \\
\hline Always & $79(63)$ & $1672(63)$ & Ref & Ref \\
\hline Sometimes or never & 47 (37) & 988 (37) & $1.0(0.7$ to 1.5$), 1.0$ & $1.0(0.7$ to 1.4$), 0.9$ \\
\hline \multicolumn{5}{|l|}{ Type of house } \\
\hline Brick & $110(89)$ & $2239(85)$ & Ref & Ref \\
\hline Semi-brick/mud & 14 (11) & $398(15)$ & $0.7(0.4$ to 1.3$), 0.2$ & $0.7(0.4$ to 1.3$), 0.3$ \\
\hline \multicolumn{5}{|l|}{ Drinking water source } \\
\hline Bottled or tap & $124(98)$ & $2624(99)$ & Ref & Ref \\
\hline Well, pump, or vendor & $2(2)$ & 37 (1) & $1.1(0.3$ to 4.8$), 0.9$ & $1.2(0.3$ to 5.0$), 0.9$ \\
\hline
\end{tabular}

*Adjusted for age and correlation within household.

†Adjusted for age only.

A study in a similar endemic setting in Bangladesh from 1966 to 1980 found cholera to be most common between 5 and 9 years of age followed by children $1-5$ years. ${ }^{11}$ Despite this and more recent hospital based reports documenting the disease in children less than 2 years of age, ${ }^{12}{ }^{13}$ World Health Organisation guidelines state that cholera should be suspected "in children over 2 years of age who have acute watery diarrhoea and severe dehydration if cholera is endemic in the local area". ${ }^{14}$ Our findings reiterate the need to change the current thinking that cholera is infrequent among children less than 2 years of age.

Healthcare utilisation patterns may have influenced the relative differences in detected cholera between age groups since the study relied on passive surveillance. In a survey included during the baseline census of the study site, young children with diarrhoea were reported to be more frequently taken for healthcare treatment compared to adults. ${ }^{5}$ It is possible that patients, particularly adults, escaped our detection system and that active surveillance could have identified more cases. ${ }^{15}$ However, it was not our intention to find all diarrhoea cases in the community, but only those perceived to be severe enough to require medical care. There are no other government outpatient centres within the study area. The project health outposts provided diagnosis, treatment of mild conditions, and transport to hospital free of charge; we gave incentives to private physicians to refer diarrhoeal cases for stool culture; the study health workers visited households to encourage them to use the outposts; and we also detected cases at the main referral hospitals. Thus, we probably captured most of the diarrhoea episodes in the community requiring medical attention.

We found that the risk factors for cholera, including having a household member with cholera detected during the study period, young age, and lower educational level are not easily amenable to intervention. It was also interesting to note that adults with cholera had a lower educational level compared to those with other diarrhoeal illnesses. The results also emphasise the high household transmission of cholera.

Improvements in living conditions could potentially prevent and ameliorate the effects not only of cholera but of diarrhoeal diseases in general. Although water points and latrines have been built and some health care has become available in the bustees during the last two and half decades, demand has far outpaced supply. Through the years, extensive subletting has resulted in overcrowding as more and more people are squeezed into available housing. ${ }^{4}$ Implementation of adequate public health infrastructure for the whole population in need may not be possible in the near future. Mass vaccination could be a potentially useful tool to prevent seasonal cholera in the area. ${ }^{16} \mathrm{~A}$ large cluster randomised efficacy trial of a killed, whole cell oral cholera vaccine is planned in Kolkata. A cluster randomised design will allow us to measure direct, as well as potential indirect (herd) protection from the vaccine ${ }^{17}$ Herd immunity against cholera would be essential in communities such as this with very crowded living conditions and where household contact is a clear risk factor for disease.

We also found extremely high rates of childhood all-cause diarrhoea in the study site, especially when it is considered that these were passively detected episodes. There are plans to explore the population rates of other diarrhoeal disease aetiologies in future studies. The site could be an ideal place to study candidate rotavirus, enterotoxigenic Escherichia coli, and Shigella vaccines for children.

\section{What is already known on this topic}

- Cholera is endemic in Kolkata with seasonal outbreaks

- The disease is known to affect all age groups

\section{What this study adds}

- The study quantifies the incidence of cholera in the study area, showing that the greatest burden is in young children

- The study identifies risk factors associated with endemic cholera in the study area, including crowding, young age, and lower educational level 
In summary, we found a substantial burden of cholera in Kolkata with risk factors not easily amenable to intervention. Young children bear the brunt not only of diarrhoeal diseases in general, but of cholera as well. Mass vaccination could be a potentially useful tool to prevent seasonal cholera in the area.

\section{ACKNOWLEDGEMENTS}

Financial support was provided by the Bill and Melinda Gates Foundation through the Diseases of Most Impoverished Program administered by the International Vaccine Institute, Seoul, Korea.

\author{
Authors' affiliations \\ D Sur, B Manna, S K Niyogi, A K Deb, S Kanungo, B L Sarkar, \\ S K Bhattacharya, National Institute of Cholera and Enteric Diseases, \\ Kolkata, India \\ J L Deen, D R Kim, M C Danovaro-Holliday, K Holliday, V K Gupta, \\ M Ali, L von Seidlein, J D Clemens, International Vaccine Institute, Seoul, \\ Korea \\ Competing interests: none

\section{REFERENCES} \\ 1 Barua D. History of cholera. In: Barua D, Greenough WB III, eds. Cholera. \\ New York: Plenum Medical Book Co, 1992:1-36. \\ 2 National Institute of Cholera and Enteric Diseases. Annual Report 2002. \\ 3 Kundu A, Nag P. Atlas of the city of Calcutta and its environs. Calcutta: \\ Ministry of Science and Technology, Government of India, 1996.
}

4 Thomas FC. Calcutta: the human face of poverty. London: Penguin Books Ltd, 1997:3-166

5 Sur D, Manna B, Deb AK, et al. Correlates for diarrhoea and treatment uptake in an impoverished slum area of Kolkata, India. J Health Popul Nutr 2004;22:130-8.

6 Smith PG, Morrow RH. Methods for field trials of interventions against tropical diseases: a toolbox. Oxford: Oxford University Press, 1991:154-311.

7 Ali M, Rasool S, Park JK, et al. Use of satellite imagery in the construction of a household GIS database for health studies in Karachi, Pakistan. Int J Health Geographics 2004;3:20.

8 Kleinbaum DG, Kupper LL, Morgenstern H. Epidemiologic research: principles and quantitative methods. New York: Van Nostrand Reinhold, 1982.

9 Newcombe RG. Interval estimation for the difference between independent proportions: comparison of eleven methods. Stat Med 1998;17:873-90.

10 Zeger SL, Liang KY. Longitudinal data analysis for discrete and continuous outcomes. Biometrics 1986;42:121-30.

11 Glass RI, Becker S, Hug MI, et al. Endemic cholera in rural Bangladesh, 19661980. Am J Epidemiol 1982;116:959-70.

12 Bhattacharya SK, Datta D, Bhattacharya MK, et al. Cholera in young children in an endemic area [letter]. Lancet 1992;340:1549.

13 Sack RB, Siddique AK, Longini IM Jr, et al. A 4-year study of the epidemiology of Vibrio cholerae in four rural areas of Bangladesh. J Infect Dis 2003:187:96-101.

14 World Health Organisation. Management of the child with a serious infection or severe malnutrition: guidelines for care at the first referral level in developing countries (WHO/FCH/CAH/OO.1). Geneva: World Health Organisation, 2000:47.

15 World Health Organisation. Making surveillance work: logistics management (WHO/V\&B/01.11). Geneva: World Health Organisation, 2001:3-7.

16 World Health Organisation Global Task Force on Cholera Control. Cholera vaccines: a new public health tool? Report of a WHO meeting, 10-11 December 2002. Geneva: World Health Organisation, 2004

17 Ukoumunne OC, Gulliford MC, Chinn S, et al. Evaluation of health interventions at area and organisation level. BMJ 1999;319:376-9.

\section{Buccal midazolam versus rectal diazepam for seizures}

- or the emergency treatment of seizures in hospital intravenous lorazepam may be used

- but in small children and outside hospital intravenous access is often difficult. Rectal

diazepam has been used for many years and more recently midazolam has been used by nasal or buccal route. Now buccal midazolam has been compared with rectal diazepam in emergency departments in Liverpool, Derby, Nottingham, and Birmingham (John McIntyre and colleagues. Lancet 2005;366:205-10; see also comment, ibid: 182-3).

The study included children aged at least 6 months who had a continuing seizure and did not have established intravenous access. A total of 219 episodes in 177 patients were included in the analysis. Randomisation at each hospital was to weekly blocks of treatment with either buccal midazolam or rectal diazepam. For each drug the dose was $2.5 \mathrm{mg}, 5 \mathrm{mg}$, $7.5 \mathrm{mg}$, and $10 \mathrm{mg}$ at ages 6-12 months, 1-4 years, 5-9 years, and 10 years or older. Most of the children $(62 \%)$ were between 1 and 4 years old (range at first treatment episode 7 months to 15 years). Treatment success (cessation of the seizure within 10 minutes without respiratory depression and with no seizure recurrence within 1 hour) was achieved in 61/109 episodes $(56 \%)$ treated with buccal midazolam and 30/110 (27\%) with rectal diazepam. (If the seizure was continuing at $10 \mathrm{~min}$ and there was intravenous access intravenous lorazepam was given.) After adjustment for hospital centre, age, diagnosis of epilepsy, presence of fever, use of antiepileptic drugs, prior treatment, and duration of seizure before treatment, buccal midazolam was significantly more effective than rectal diazepam (odds ratio 4.1). The median time to seizure cessation was 8 min (midazolam) vs $15 \mathrm{~min}$ (diazepam). Intravenous lorazepam was used in 36 episodes $(33 \%)$ treated with midazolam and 63 episodes (57\%) treated with diazepam. Respiratory depression was recorded in five episodes in the midazolam group and seven in the diazepam group.

Buccal midazolam is more effective than rectal diazepam for the emergency treatment of children with seizures. 